\title{
Suppression of Pressure Oscillations for Supersonic Cavity Flow with Passive Control
}

\author{
Chao ZHANG ${ }^{a}$, De-Jun SUN ${ }^{\text {b, }}$, Zhen-Hua WAN ${ }^{c}$ \\ ${ }^{1}$ Department of Modern Mechanics, University of Science and Technology of China, Hefei 230027, \\ China \\ azzzhchao@mail.ustc.edu.cn, ${ }^{b}$ dsun@ustc.edu.cn, cwanzh@ustc.edu.cn \\ ${ }^{*}$ Corresponding author
}

\begin{abstract}
Keywords: Large Eddy Simulation (LES), Dynamic Mode Decomposition (DMD), Supersonic Cavity Flow, Pressure Oscillation.
\end{abstract}

\begin{abstract}
Large eddy simulation (LES) for a supersonic Mach 1.8 flow past 2D cavities with/without passive control is carried out to investigate the characteristics of oscillations, and the dynamic mode decomposition (DMD) is used to identify the dominant flow structures with pertinent frequencies. A passive control by substituting the cavity trailing edge with a quarter-circle is studied. The pressure field for the dominant dynamic mode becomes more uniform, the intensity of fluctuations in the shear-layer is reduced, and the wavelength of the shear-layer vortices is significantly shortened under control. It is found that the dominant oscillation frequency of the cavity corresponds to the third Rossiter mode, which is significantly increased after control. The amplitude of the pressure oscillations in the cavity shifts to lower level. The sound pressure level (SPL) can even be reduced by approximate $10 \mathrm{~dB}$. The oscillation mechanism illustrated by the DMD remains the same after passive control. Overall, the oscillations of the cavity flow are closely linked with the shear-layer instability, which is suppressed by the current control technique.
\end{abstract}

\section{Introduction}

In the past decades, cavity flows have received great attention in both experimental and numerical investigations due to its relevance to many practical engineering applications. The oscillations are typically described as a flow-acoustic resonance phenomenon. Its first detailed description is credited to Rossiter [1], a semi-empirical formula is developed based on the feed-back mechanism to predict the resonant frequencies. Heller \& Bliss [2] modified the formula. As Rowley \& Williams [3] mentioned, the feedback mechanism contains only four elemental fluid dynamic processes: (1) shear layer amplification of vortical disturbances; (2) pressure wave generation through vortex-surface interaction; (3) upstream propagation of acoustic waves; (4) receptivity at the front wall of the cavity and converting pressure waves into vorticity waves.

Much research work about control of cavity flow oscillations has been conducted. For passive control, the common method is to mount spoilers [4] at the upstream of the cavity front wall or replace the cavity front/rear wall by ramps [5]. It has proven to be effective that using a sub-cavity on the front wall of the cavity covered by a flat plate can suppress the supersonic flow oscillations [6,7]. However, the effectiveness of passive techniques relies on specified flow conditions[3]. For active open-loop control, the mass injection is frequently utilized at the cavity front wall or the upstream of it [8,9], but effective active open-loop methods often require large actuator power [3]. For supersonic cavity flow, the existing research results show that passive control and active open-loop control can be achieved, while active closed-loop control has not been used until now.

The flow complexity may be reduced to a set of coherent features with a few characteristic structures possessing self-excited global modes in space and time. Proper orthogonal decomposition (POD) has been extensively used to identify the coherent structures in turbulent flows. Schmid [10] introduced dynamic mode decomposition (DMD) approach to extract dynamic mode information from a flow field based on the Koopman analysis of dynamical system. DMD attempts to represent a data sequence by 
orthogonalizing it in time, while POD attempts a decomposition based on orthogonality in space. Furthermore, the DMD is directly applied to the data, while a POD analysis processes second-order statistics of the data.

In the present investigation, a two-dimensional open cavity of length-to-depth ratio $\mathrm{L} / \mathrm{D}=4$ has been studied at Mach 1.8, and then we investigate a passive control technique by substituting the cavity trailing edge with a quarter-circle. The coherent structures of the flow field with/without current control are identified using the DMD approach.

\section{Numerical Methods}

The schematic of the two-dimensional open cavity model with/without the passive control is shown in Fig. 1. First, a rectangular baseline cavity (named cavity R0) is installed in a rectangular duct with a height $(H)$ of $20 \mathrm{~cm}$. The cavity has a depth $(D)$ of $5 \mathrm{~cm}$, its length $(L)$ is given as $4 D$. The Reynolds number based on the cavity depth $(D)$ and the acoustic speed is $2.5 \times 10^{5}$ approximately, the Sutherland viscosity law is employed in the computations. The Mach number of the free stream flow is 1.8 . Then, a passive control by substituting the trailing edge with a quarter-circle is studied, with the radius $(R)$ equals $D$ (named cavity R5). The origin of the coordinate is located at the leading edge. Monitors of $P_{1}$ and $P_{4}$ are located at the middle of the floor and the front wall, the other two monitors of $P_{2}$ and $P_{3}$ are located around the leading edge $(x / D=-0.2, y / D=0)$ and the trailing edge $(x / D=5.2, y / D=0)$. During the whole computation, local wall static pressure is recorded at these monitors.

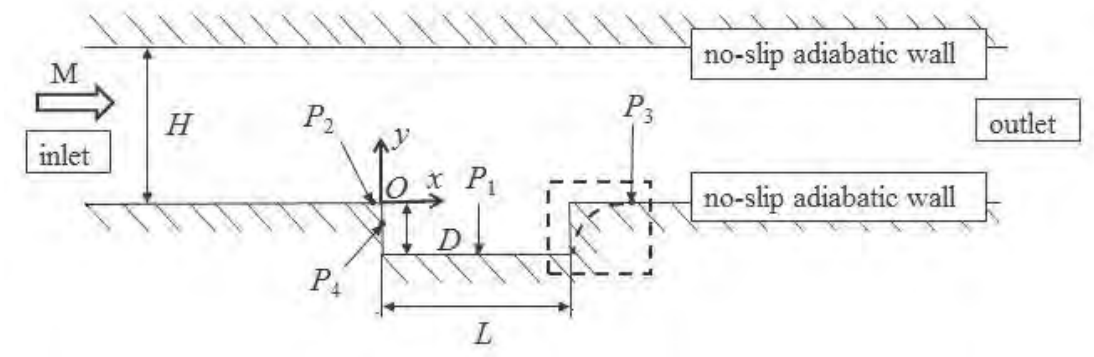

Fig.1 Schematic of the Cavity Model with/without the Passive Control (shown in the dashed rectangle)

In the computation, an implicit second-order upwind Roe-FDS scheme is used for spatial discretization. The time integration is performed by a second-order implicit approach, the inner iteration of which is achieved by a Gauss-Seidel method. LES using the Smagorinsky-Lilly model has been performed, and the initial conditions are obtained from a steady Raynolds Averaged Navier-Stokes (RANS) computation with Spalart-Allmaras turbulence model.

A non-uniform structural grid is used with the mesh points clustered near the wall. The upper cavity region is built up by $640 \times 200$ rectangular cells, and $200 \times 200$ cells are used inside cavity region. The supersonic inflow boundary, namely the physical boundary conditions $(M=1.8, p=0.1 \mathrm{MPa}, T=600 \mathrm{~K})$ are applied at the inlet boundary. No-slip and adiabatic conditions are applied on the solid walls. Extrapolated boundary is utilized for the outlet boundary. For the unsteady integration, a small time-step of $1.0 \times 10^{-6} \mathrm{~s}$ is employed for all cases.

The DMD algorithm is applied in a subdomain to identify the coherent structures of the cavity flows. The subdomain consists of the rectangle region $(x / D, y / D) \in[-2,6] \times[0,2]$ and the inside cavity region. In our computations, 200 instantaneous snapshots of the unsteady flow fields are used. According to the Nyquist criterion [10], we use a temporal separation of $20 \mu \mathrm{s}$ for the snapshots.

Simulations of 2D supersonic $(M=1.83)$ cavity models with $L / D=1$ and $L / D=2.2(D=12 \mathrm{~mm})$ are chosen to validate the present numerical method. Fig. 2 shows the Strouhal numbers $S t$ for rectangular cavities with different $L / D$. Empty circle (black color) represents the previous experimental results referred by Alam et.al. [6]. Solid circle (black color) represents the computation results of Alam et.al. [6]. Broken and solid lines (black color) represent the results of Heller \& Bliss [2] and Nishioka et.al. [11], respectively. Solid diamond (red color) represents our results. The comparison shows that our results are close to those obtained by Alam et.al. [6] For $L / D=1$ cavity, and the results for $L / D=2.2$ cavity are close 
to the previous experimental results. Both simulated results are close to the results obtained by Nishioka et.al. [11].

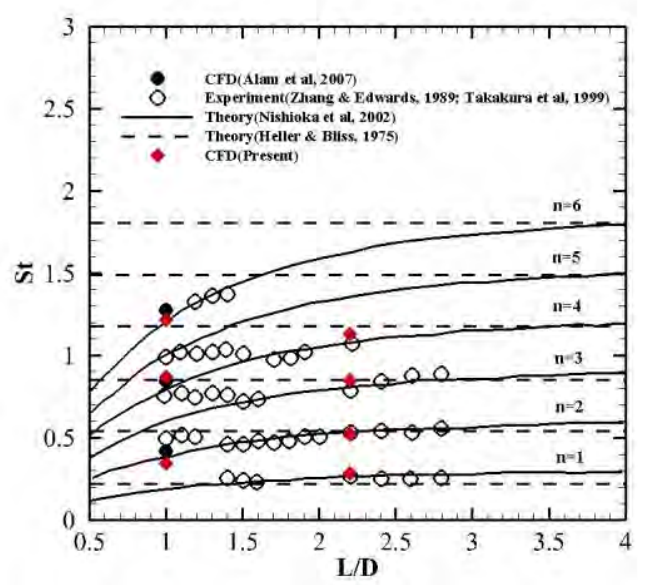

Fig. 2 Strouhal Numbers $S t$ for Rectangular Cavities with Different L/D (M=1.83)

\section{Results and Discussions}

As aforementioned, Heller \& Bliss [2] developed the modified Rossiter's semi-empirical formula to predict the resonant frequencies as

$$
f_{m}=\frac{u_{\infty}}{L} \frac{m-\alpha}{M_{\infty} / \sqrt{1+\left[\left(\gamma_{\infty}-1\right) / 2\right] M_{\infty}^{2}}+1 / \mathrm{K}_{v}}
$$

where $f_{m}$ is the frequency at a given mode number $m, u_{\infty}$ is free stream velocity, $L$ is the cavity length, $M_{\infty}$ is free stream Mach number, $\gamma_{\infty}$ is the ratio of specific heats, $\mathrm{K}_{v}=0.57$ and $\alpha=0.25$ are empirical constants. Based on the present flow conditions, as predicted by Eq. (1), the first three resonant frequencies are $1050 \mathrm{~Hz}, 2450 \mathrm{~Hz}$ and $3850 \mathrm{~Hz}$, respectively.

Fig. 3(a) and (b) show the pressure histories and their spectra for the four monitors for the baseline cavity. The amplitude of the pressure fluctuation as well as the PSD (with a unit of $\mathrm{Pa}^{2} / \mathrm{Hz}$ ) is higher in the cavity region than outside the cavity region. The dominant oscillation frequency is $3787 \mathrm{~Hz}$, corresponding to the third Rossiter mode.

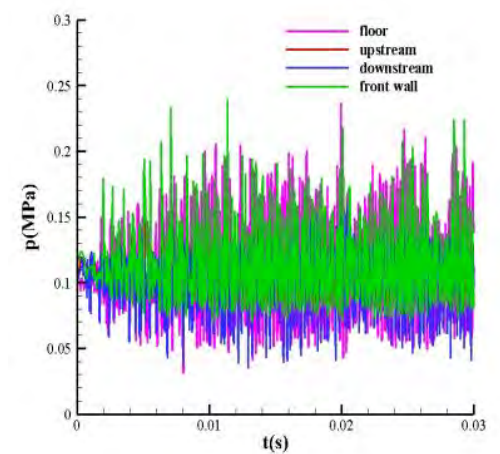

(a)

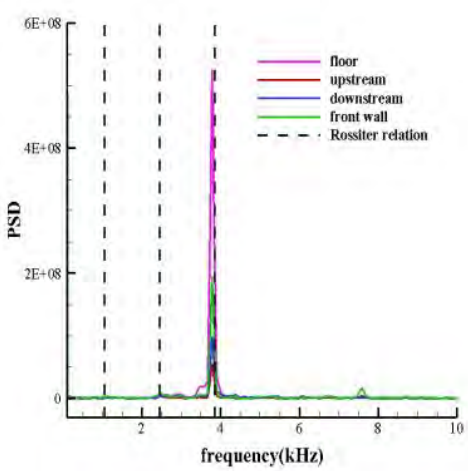

(b)

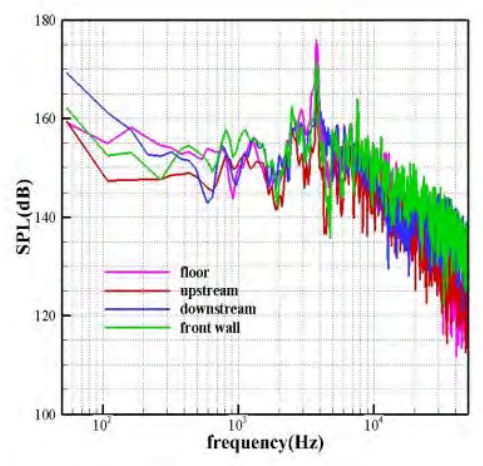

(c)

(b) FFT results

(c) SPL results

Fig. 3 Pressure Histories, FFT Results and SPL Results of the Four Monitors for the Baseline Cavity by

The sound pressure level (SPL) is obtained from PSD as a function of frequency [12], which is defined 


$$
S P L=10 \log _{10}\left(\frac{P S D}{p_{r e f}^{2}}\right)
$$

where $p_{\text {ref }}=2 \times 10^{-5} \mathrm{~Pa}$ is the reference pressure, represents the value adopted as the minimum audible sound pressure variation that propagates in gases.

The SPLs of the four monitors for the baseline cavity are shown in Fig. 3(c). Inside the cavity, the maximum value of SPL is around $176 \mathrm{~dB}$ at monitor $P_{1}$, and $172 \mathrm{~dB}$ at monitor $P_{4}$. Outside the cavity, the corresponding value is $164 \mathrm{~dB}$ at monitor $P_{2}$ and $168 \mathrm{~dB}$ at monitor $P_{3}$. Previous results have shown that the small region around the trailing edge can be seen as a tone source; the SPL becomes smaller as the distance away from the tone source becomes farther. Our simulations seem to support this contention.

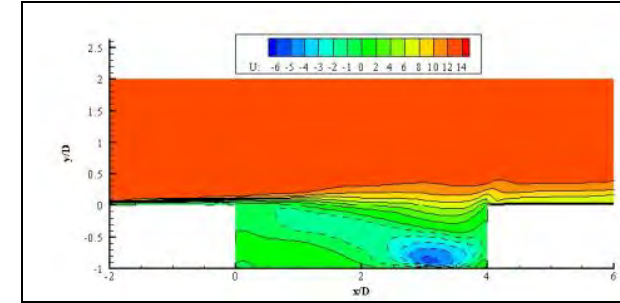

(a)

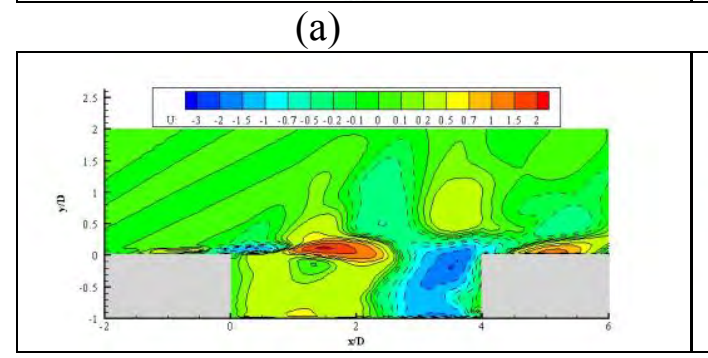

(c)

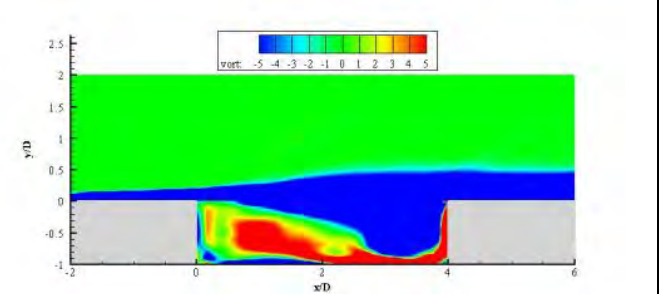

(b)

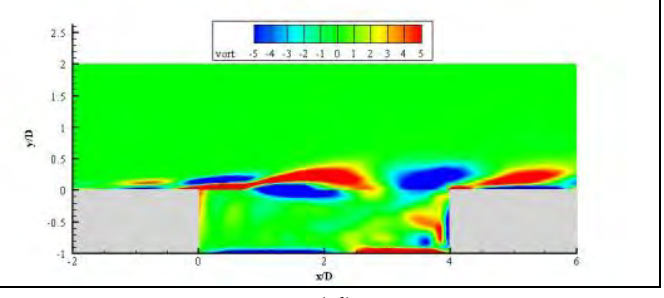

(d)

(a) (b) the time-averaged dynamic mode

(c) (d) the dominant oscillation dynamic mode

Fig. 4 Representative Dynamic Modes (real part only), Visualized by the Streamwise Velocity Component [left] and the Vorticity [right]

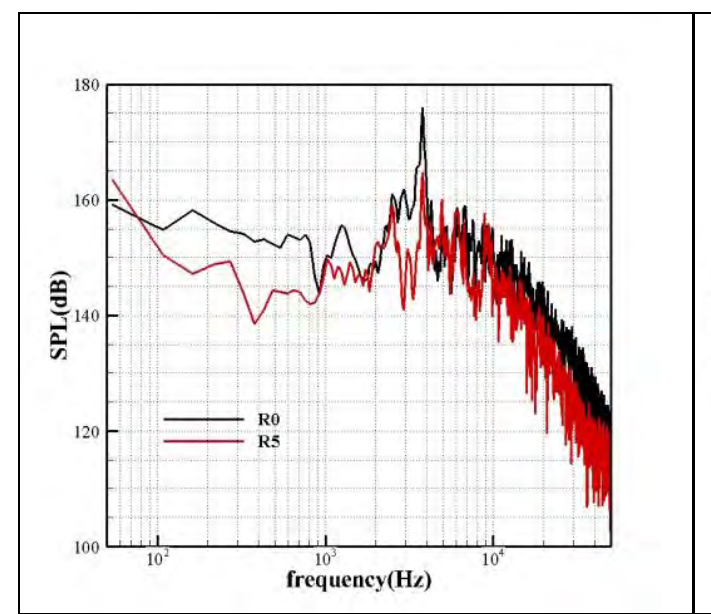

(a) SPL results at monitor $P_{1}$

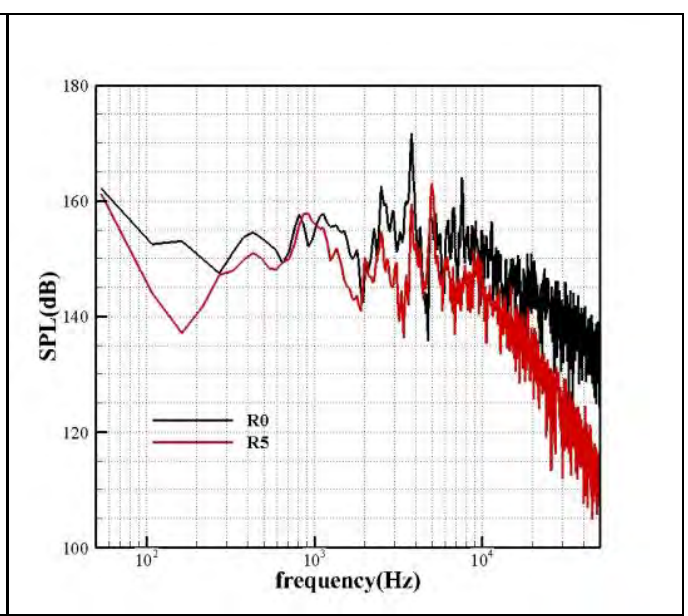

(b) SPL results at monitor $P_{4}$

Fig. 5 SPL Results of the Two Monitors in the Cavity Region with/without Passive Control

Based on the unsteady flow fields, we can get the dominant flow structures given by DMD. From the energy spectrum (not shown in the paper), the oscillation frequencies corresponding to the first three Rossiter modes are $3804 \mathrm{~Hz}, 2701 \mathrm{~Hz}$ and $1409 \mathrm{~Hz}$. These three dynamic modes marked as mode 1, 2 and 3 , respectively. The dynamic mode with oscillation frequency of 0 marked as mode 0 , corresponding to the time-averaged flow. Fig. 4 shows mode 0 and mode 1 (real part only) using the streamwise velocity component (normalized with the free stream) and the vorticity (normalized with the free stream and the 
depth of the cavity). For the time-averaged flow, a large recirculation region closed to the rear wall can be seen. For the dominant oscillation dynamic mode, the flow field displays a clear shear layer structure. Due to the Kelvin-Helmholtz instability, the large-scale vortices are generated [1] and their sizes would grow slowly along the shear layer.

It is well known that the cavity trailing edge plays an important role in the feedback process of the oscillation; hence, it would be natural to consider that we can break or weaken such feedback process by just modifying the shape of this trailing edge. Here, we substitute the trailing edge with a quarter-circle, where the radius equals $D$. Fig. 5 shows the SPLs of the two monitors $\left(P_{1}\right.$ and $\left.P_{4}\right)$ inside the cavity region with/without control, one can see that the SPL corresponding to the dominant oscillation frequency in the cavity is reduced by even up to $10 \mathrm{~dB}$. Also, there is a decreasing trend for the SPL at high frequency. The above results demonstrate that the current control methodology is quite effective.

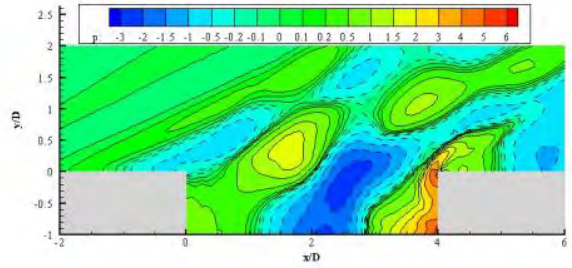

(a)

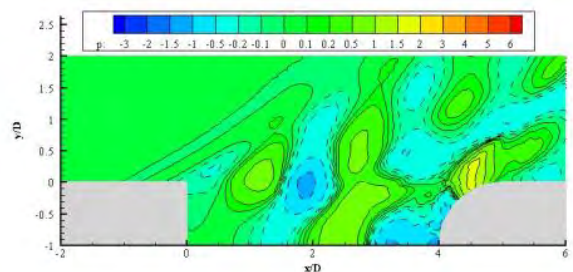

(b)

(a) (b) visualized by the pressure

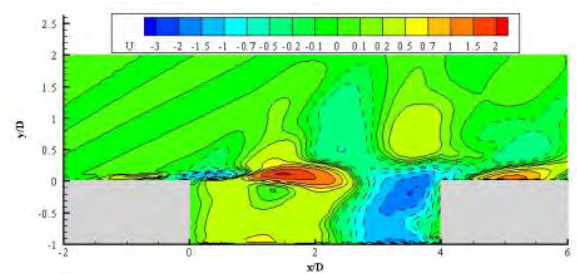

(c)

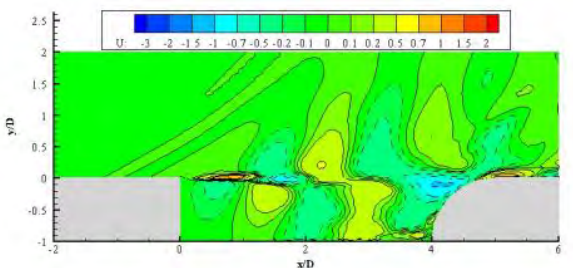

(d)

(c) (d) visualized by the streamwise velocity component

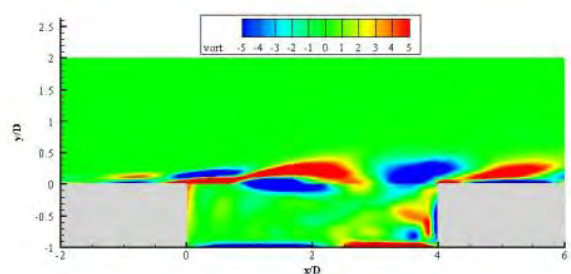

(e)

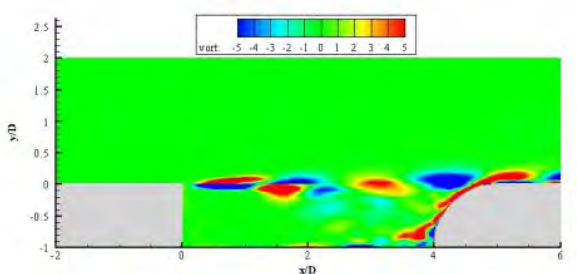

(f)

(e) (f) visualized by the vorticity

Fig. 6 The Dominant Oscillation Dynamic Modes (real part only) without [left] and with [right] Passive Control

The DMD results show that the dominant oscillation frequency for cavity $\mathrm{R} 5$ is $5125 \mathrm{~Hz}$ (not shown in the paper), significantly increased compared with cavity R0 $(3804 \mathrm{~Hz})$. Fig. 6(a) and (b) show the dominant oscillation dynamic modes (real part only) visualized by the pressure (normalized with the static pressure) with/without passive control. Intuitively, the pressure field becomes more uniform under the passive control, which explains why the amplitude of the pressure fluctuations as well as the SPL corresponding to the dominant oscillation frequency shifts to lower levels. The dominant oscillation dynamic modes (real part only) visualized by the streamwise velocity component and the vorticity are shown from Fig. 6 (c) to (f), the flow fields under passive control display clear shear layer structures as well, indicating that the oscillation mechanism is unchanged. But, the intensity of fluctuations in the shear-layer is reduced. The wavelength of the shear-layer vortices for cavity R5 is significantly shortened than that of the cavity R0. 


\section{Conclusion}

The present study investigates the characteristics of oscillations in 2D supersonic cavity flows with/without passive control by LES, and analyzes the flow structures by DMD. Under current flow conditions, the dominant oscillation frequency is corresponding to the third Rossiter mode. The SPL can even be reduced by approximate $10 \mathrm{~dB}$ with a passive control by substituting the cavity trailing edge with a quarter-circle. DMD results show that the pressure field becomes more uniform under control. The dominant oscillation dynamic modes display clear shear layer structures with/without the passive control, indicating that the oscillation mechanism is unchanged. But, the intensity of fluctuations in the shear-layer is reduced. The wavelength of the shear-layer vortices is significantly shortened under control. Overall, the oscillations of the cavity flow are closely linked with the shear-layer instability, which is suppressed by the current control technique.

\section{Acknowledgement}

This work is supported by Nation Natural Science Foundation of China (Grant Nos. 11232011, 11402262). The author Z. Wan thanks China Postdoctoral Science Foundation funded project (Grant No. 2014M561833).

\section{References}

[1] J. E. Rossiter, Wind-tunnel experiments on the flow over rectangular cavities at subsonic and transonic speeds, Aero. Res. Counc. R \& M., (1964).

[2]H. H. Heller, D. B. Bliss, The physical mechanism of flow induced pressure fluctuations in cavities and concepts for their suppression, AIAA Paper, (1975).

[3]C. W. Rowley, D. R. Williams, Dynamics and control of high Reynolds number flow over open cavities, Annu. Rev. Fluid Mech, 38 (2006), 251-276.

[4]J. G. Dudley, L. Ukeiley, Detached eddy simulation of a supersonic cavity flow with and without passive flow control, AIAA, (2011).

[5]N. S. Vikramaditya, J. Kurian, Experimental study of influence of trailing wall geometry on cavity oscillations in supersonic flow, Experimental Thermal and Fluid Science, 54 (2014), 102-109.

[6]Md. M. Alam, S. Matsuo, K. Teramoto, T. Setoguchi, H. D. Kim, A new method of controlling cavity-induced pressure oscillations using sub-cavity, Journal of Mechanical Science and Technology, 21 (2007), 1398-1407.

[7]Y. K. Lee, M. S. Kang, H. D. Kim, T. Setoguchi, Passive control techniques to alleviate supersonic cavity flow oscillation, Journal of Propulsion and Power, 24-4 (2008), 697-703.

[8]T. Lusk, L. Cattafesta, L. Ukeiley, Leading edge slot blowing on an open cavity in supersonic flow, Exp Fluids, 53 (2012), 187-199.

[9]W. P. Li, T. Nonomura, K. Fujii, Mechanism of controlling supersonic cavity oscillations using upstream mass injection, Phys. Fluids, 25 (2013) .

[10]P. J. Schmid, Dynamic mode decomposition of numerical and experimental data, J. Fluid Mech, 656(2010), 5-25.

[11]M. Nishioka, T. Asai, S. Sakaue, K. Shirai, Some thoughts on the mechanism of supersonic cavity flow oscillation, Part 2 A new formula for the oscillation frequency. Journal of Japan Society of Fluid Mechanics, 21 (2002).

[12]Shia-Hui Peng, Simulation of turbulent flow past a rectangular open cavity using DES and unsteady RANS. AIAA, (2006). 